\title{
Modeling and forecasting solar energetic particle events at Mars: the event on 6 March 1989
}

\author{
A. $\operatorname{Aran}^{1}$, D. Lario ${ }^{2}$, B. Sanahuja ${ }^{1,3}$, R. G. Marsden ${ }^{4}$, M. Dryer ${ }^{5,6}$, C. D. Fry ${ }^{6}$, and S. M. P. McKenna-Lawlor ${ }^{7}$ \\ 1 Department d'Astronomia i Meteorologia, Universitat de Barcelona, 08028 Barcelona, Spain \\ e-mail: aaran@am.ub.es \\ 2 The Johns Hopkins University, Applied Physics Laboratory, Laurel, MD 20723, USA \\ 3 Institut d'Estudis Espacials de Catalunya (IEEC-ICC), 08028 Barcelona, Spain \\ 4 Research and Scientific Support Department of European Space Agency, ESTEC, Noordwijk 2201AG, The Netherlands \\ 5 Space Environment Center, National Oceanic and Atmospheric Administration, Boulder, CO 80305, USA \\ ${ }^{6}$ Exploration Physics International, Inc., Huntsville, AL 35806, USA \\ 7 Space Technology Ireland, National University of Ireland, Maynooth, Ireland
}

Received 2 February 2007 / Accepted 28 March 2007

\begin{abstract}
Context. Large solar energetic particle events are able to enhance the radiation intensity present in interplanetary space by several orders of magnitude. Therefore the study, modeling and prediction of these events is a key factor to understand our space environment and to protect manned space missions from hazardous radiation.

Aims. We model an intense solar energetic particle event observed simultaneously on the 6 of March 1989 by the near-Earth orbiting spacecraft IMP-8 and by the Phobos- 2 spacecraft in orbit around Mars (located $72^{\circ}$ to the East of the Earth and at 1.58 AU from the Sun). This particle event was associated with the second largest X-ray flare in solar cycle 22. The site of this long-duration X15/3B solar flare was N35E69 (as seen from the Earth) and the onset of the 1-8 A X-ray emission occurred at 1350 UT on 6 March 1989. A traveling interplanetary shock accompanied with $<15 \mathrm{MeV}$ proton intensity enhancements was observed by IMP-8 at 1800 UT on 8 March and by Phobos-2 at 2015 UT on 9 March. This shock determines the particle intensities at both spacecraft.

Methods. We use an MHD code to model the propagation of the associated shock to both spacecraft and a particle transport code to model the proton intensities measured by IMP-8 and Phobos-2. By assuming that energetic particles are continuously accelerated by the traveling shock, and that the injection rate of these particles, $Q$, into the interplanetary medium is related to the upstream-todownstream velocity ratio, $V R$, at the point of the shock front that connects with the observer, we perform predictions of the solar energetic particle intensities observed at Mars from those measured at Earth.

Results. We reproduce not only the arrival times of the shock at both spacecraft but also the measured jump discontinuity of solar wind speed, density and magnetic field. Also, we reproduce the $0.5-20 \mathrm{MeV}$ proton intensities measured by both spacecraft. Functional dependences such as the $Q(V R)$ relation deduced here allow us to predict the proton intensities measured at Phobos- 2 for this event. Applications of this model for future predictions of solar energetic particle fluxes at Mars are discussed.
\end{abstract}

Key words. Sun: solar-terrestrial relations - Sun: particle emission - shock waves - Sun: coronal mass ejections (CMEs)

\section{Introduction}

Large solar energetic particle (SEP) events are an important element in assessing the radiation hazards in space. The objective of sending humans to Mars must be accompanied by basic protection rules (Lanzerotti 2004). To ensure astronaut safety, it is important to know how intense an episode of enhanced radiation will be; when it will reach dangerous levels; when its maximum intensity will be attained, and how long it will last. In terms of SEPs, all these parameters depend on the location, speed, strength and extent of the energetic particle source relative to the observer, as well as on the complexity of the heliospheric magnetic field at the time of the SEP event (Turner 2000). At present, there are few observational analyses on the dependence of particle flux and fluences with radial distance (a summary can be found in Lario et al. 2006). Recommendations for radial extrapolation of SEP intensities from measurements at 1.0 AU (Feynman \& Gabriel 1988) have been proven to be unsuccessful, especially when traveling interplanetary shocks dominate the evolution of the particle intensity time profiles (Smart \& Shea 2003). To our knowledge no studies dealing with forecasting individual SEP events at Mars orbit from SEP Earthorbit observations have been reported.

Our understanding of the physics involved in the development of large SEP events is mainly based on observations at 1.0 AU and it assumes that the dominant source of energetic particles is the shock driven by coronal mass ejections (CMEs) (Kahler 2001). When a fast CME occurs, it may be able to drive a shock wave ahead of it that accelerates particles as it moves through both the solar corona and interplanetary space (Cane et al. 1988). Energetic particles stream out along the interplanetary magnetic field (IMF) lines reaching observers located in the heliosphere. As the CME-driven shock expands, it may continuously accelerate particles as it propagates through interplanetary space and thus be able to fill broad regions of the inner heliosphere with SEPs (Cliver et al. 1995).

In order to model large SEP events we need to determine how particles and shocks propagate through the interplanetary medium, and how shocks accelerate and inject particles into interplanetary space as they move out from the Sun. Several models have appeared in the literature that approximate in 
different ways the series of complex and diverse phenomena occurring during the development of SEP events. A summary of these models can be found in the works of Lario (2005) and Lee (2005). In this work we follow the model developed by Lario et al. (1998). We use a 2.5-dimensional magnetohydrodynamic (MHD) model (Smith \& Dryer 1990) to simulate the expansion and propagation of the associated interplanetary shock from 0.08 to $2 \mathrm{AU}$, reproducing both the arrival time of the shock and the jump of the solar wind parameters measured by spacecraft in the interplanetary medium. We also solve the focused-diffusion transport equation (Ruffolo 1995) to describe the propagation of energetic particles along the IMF lines. In order to model the observed SEP fluxes, we assume that shock-accelerated particles are injected onto the IMF lines at the point of the shock front that magnetically connects with the observer. We do not treat the fundamental nature of particle acceleration at the traveling interplanetary shock, but we consider the shock acceleration as an ad hoc source term, $Q(t, r)$, that represents the injection rate of shock-accelerated particles at the time $t$ and at the heliocentric radial distance $r$. Energetic particles propagate along the IMF lines undergoing the effects of pitch-angle scattering, solar wind convection and adiabatic deceleration (Ruffolo 1995). The topology of the field lines upstream of the traveling shock is considered to be a nominal Parker spiral with a prescribed value of the solar wind speed. Details of the model and its applicability to space weather operations can be found, respectively, in the works of Lario et al. (1998) and Aran et al. (2005).

The simulation of shock-associated SEP events observed simultaneously by different spacecraft in the ecliptic plane requires the use of at least 2-dimensional models of shock propagation. The longitudinal dependence of the SEP intensity-time profiles simultaneously observed by these spacecraft is only reproducible if the magnetic connection between the shock and the observer is correctly described through, for example, a Parker spiral magnetic field but not with the assumption of a radial magnetic field. The assumption that a specific shock-acceleration particle mechanism works at the traveling shock is an oversimplification in view of the rich diversity of events observed in association with the passage of interplanetary shocks (van Nes et al. 1984; Tsurutani \& Lin 1985; Lario et al. 2003a). Variables that describe particle injection into the shock-acceleration mechanisms and particle escape from the turbulent medium formed in the vicinity of the shock increase the number of free parameters to use in the models of SEP events. For these reasons we have preferred to use a simple model (i.e. Lario et al. 1998), which does not explicitly consider a shock-acceleration mechanism, leaving the use of more elaborated models for the future when a better understanding of the physical conditions at the acceleration site will be achieved.

We apply this model to one of the few SEP events that, fortunately, was observed by spacecraft orbiting around the Earth and by spacecraft orbiting around Mars (Marsden et al. 1990; McKenna-Lawlor et al. 1991, 2005). For this event, the upstream solar wind had not been disturbed by any former interplanetary transient event. While the observation of SEP events at the orbit of the Earth has been routinely performed for the last three solar cycles (Lario \& Simnett 2004, and references therein), the observation of SEP events at Mars has only been possible in a few rare occasions. Missions such as Phobos- 2 and Mars-Odyssey have offered us only a slight clue of the SEP environment at Mars (e.g. Marsden et al. 1991; McKenna-Lawlor et al. 1991; Cleghorn et al. 2004).

In this paper we use energetic particle data from the Low Energy Telescope (LET) on board Phobos-2
(Marsden et al. 1990) and the Charged Particle Measurement Experiment (CPME) on board IMP-8 (Sarris et al. 1976) to compare the SEP environment at the Earth and Mars during an intense SEP event of solar cycle 22. Phobos-2 was launched on 12 July 1988 and reached Mars on 29 January 1989. It was inserted into orbit around Mars and, after several orbit corrections, it was lost on 27 March 1989 due to a failure of the on board control electronics (Sagdeev \& Zakharov 1989). Therefore, the opportunity to study this particle event is quite unique even though this is not a text-book case for modeling: Phobos-2 particle data contains a relevant gap after the onset of the SEP event and there are no particle anisotropy measurements. In addition, the accuracy of the solar wind data available is low and there is a lack of useful observations of the IMF because of the orbit of Phobos- 2 around Mars.

In Sect. 2 we present the characteristics of this event as seen by IMP-8 and Phobos-2. In Sect. 3 we apply the shock-andparticle model to describe the SEP event measured by each of the two spacecraft. We discuss the predictions of SEP profiles in the Martian environment in Sect. 4 and the predicted maximum particle intensities and fluences in Sect. 5. Finally, we give a discussion and conclusions in Sect. 6.

\section{The solar energetic particle event on 6 March 1989}

The SEP event analyzed in this paper was the first in a series of events detected in March 1989 (McKenna-Lawlor et al. 2005). The transit of the NOAA Active Region 5395 over the solar disk was marked by an intense level of solar activity including the occurrence of at least $10 \mathrm{X}$-class X-ray flares (Feynman $\&$ Hundhausen 1994). This large and complex active region rotated onto the visible disk of the Sun (as seen from the Earth) on 6 March 1989. This same day the second largest X-ray flare in solar cycle 22 (estimated to be a class X15) occurred (Watari et al. 2001). An optical 3B class flare took place at N35E69 with onset at $1354 \mathrm{UT}$. The 1-8 $\AA$ X-ray emission began at $1350 \mathrm{UT}$ and reached maximum flux at $1405 \mathrm{UT}$. A fast coronal mass ejection (CME) was seen by the Solar Maximum Mission (SMM) over the northeast limb of the Sun at 1415 UT (Feynman $\&$ Hundhausen 1994). At the time of the X15 flare on 6 March 1989 , Phobos- 2 was in circular equatorial orbit at an altitude of $\sim 2$ Martian radii, and located $72^{\circ}$ to the East of the Earth at a heliocentric distance of 1.58 AU (McKenna-Lawlor et al. 2005). We have limited this study to the first SEP event because only before the arrival of the first interplanetary shock at both spacecraft was the solar wind stable enough to allow a reasonable modeling of the existing upstream interplanetary conditions, both for shock propagation and interplanetary magnetic field topology.

Several authors (e.g. Marsden et al. 1990; Kurt et al. 2004) have associated the SEP event observed by IMP-8 on 6 of March and later by Phobos-2, with the X15/3B flare at N35E69. We agree with this identification (see below), in spite that McKenna-Lawlor et al. (2005) associated the particle intensity enhancement at IMP-8 with a C5.2/SF flare that occurred at 2356 UT on 5 March from S20W61 (see their paragraph [25]). Figure 1 of McKenna-Lawlor et al. (2005) shows that 4.6-15.0 MeV proton intensities at IMP-8 started to increase above the background at $\sim 2000$ UT on 6 March, whereas the 48-96 MeV proton intensity enhancement at IMP-8 was not detected until the end of a data gap at $\sim 1200$ UT on 7 March. The nominal Parker spiral magnetic field connection between IMP-8 and the Sun implies that the site of the C5.2/SF flare was well 
connected to IMP-8. However, SEP events originating from activity at western longitudes have rapid intensity rises shortly after the occurrence of the solar flare (Cane et al. 1988), whereas in the case of this event the intensity enhancement was gradual and with a significant delay with respect to the parent solar event as typically observed in eastern events. In addition, the low-energy proton intensities measured by IMP-8 started to increase earlier than the higher energy intensities contrary to what is expected from well-connected western events. Although the correlation found between solar flare $\mathrm{X}$-ray integrated fluxes and peak fluxes of $>10 \mathrm{MeV}$ protons is weak (cf. Fig. 1 of Balch 1999), it seems reasonable to expect that the solar event associated with an X15/3B flare was more productive in terms of energetic particles than the events associated with the C5.2/SF flare. Hence, we will assume that this X15/3B flare was temporally associated with the SEP event observed by both spacecraft.

IMP-8 observed the passage of an interplanetary shock at $\sim 1800$ UT on 8 March with a local increase of the low-energy $(<15 \mathrm{MeV})$ proton intensities. The passage of this shock was associated with the occurrence of a Sudden Storm Commencement (SSC) at 1755 UT on 8 March (Marsden et al. 1990) and a decrease of the Dst index to $\sim-100 \mathrm{nT}$. A later SSC was also observed at the Earth at 1900 UT on 9 March, and the Dst that was already low, decreased again to $-100 \mathrm{nT}$. McKenna-Lawlor et al. (2005) attributed the SSC on March 9 to the solar event associated with the $\mathrm{X} 15 / 3 \mathrm{~B}$. This association implies an average transit speed for the associated plasma disturbance to travel from the Sun to the Earth of $\langle v\rangle=539 \mathrm{~km} \mathrm{~s}^{-1}$. According to these authors the shock observed by IMP-8 at 1800 UT on 8 March was probably originated during the C5.2/SF flare, implying an average transit speed for the associated solar wind disturbance of $\langle v\rangle=629 \mathrm{~km} \mathrm{~s}^{-1}$.

Fast CMEs tend to occur in association with intense solar flares (Feynman \& Hundhausen 1994; Dryer 1996; Gopalswamy et al. 2004), therefore, it is reasonable to assume that the CME that occurred in temporal association with the X15/3B was faster than the presumed CME associated with the C5.2/SF (if any). Since the longitudinal separation between the Earth and the sites of the X15/3B and C5.2/SF flares were similar (E69 and W61, respectively), it is reasonable to assume that the shock associated with the X15/3B flare was faster and had stronger effects on the Earth's environment than the presumed shock associated with the C5.2/SF flare. In view of all these facts, we consider that the shock at 1800 UT on 8 March at IMP- 8 was originated during the solar event associated with the X15/3B flare. By using this association we deduce that the time interval between the occurrence of the solar flare and the shock passage at $1 \mathrm{AU}$ is $52.1 \mathrm{~h}$ implying an average transit speed of $\langle v\rangle=798 \mathrm{~km} \mathrm{~s}^{-1}$.

Marsden et al. (1990) identified the passage of a shock at Phobos-2 at 2015 UT on 9 March, in coincidence (within the precision of the solar wind data available) with the highest flux values of a SEP event measured by the Phobos-2/LET instrument. According to these authors, this shock originated from the solar events occurring in association with the X15/3B flare. The time interval between the occurrence of this solar flare and the shock passage at Phobos-2 was $78.4 \mathrm{~h}$, thus implying an average transit speed from the Sun to $1.58 \mathrm{AU}$ of $\langle v\rangle=837 \mathrm{~km} \mathrm{~s}^{-1}$. The longitudinal separation between the site of the solar flare and the Phobos- 2 location was only $3^{\circ}$. Because of the motion of Mars, the longitudinal separation between the location of Mars on 9 March and the site of the X15/3B flare on 6 March was only $2^{\circ}$. The assumption made by McKenna-Lawlor et al. (2005) that the shock originated at the time of the X15/3B flare reached IMP-8 at $\sim 1900$ UT on 9 March and Phobos-2 at $\sim 2015$ UT on
9 March implies a shock that moves very fast in the direction toward Mars $\left(\langle v\rangle=837 \mathrm{~km} \mathrm{~s}^{-1}\right)$ but extremely slowly toward the Earth $\left(\langle v\rangle=539 \mathrm{~km} \mathrm{~s}^{-1}\right)$. We argue that the shock originated in association with the X15/3B flare had an approximate uniform radial expansion over at least $72^{\circ}$ in longitude being able to reach IMP-8 at 1800 UT on 8 March $\left(\langle v\rangle=798 \mathrm{~km} \mathrm{~s}^{-1}\right)$ and Phobos-2 at 2015 UT on 9 March $\left(\langle v\rangle=837 \mathrm{~km} \mathrm{~s}^{-1}\right)$. Therefore, we consider that the solar event associated with the X15/3B flare generated not only the SEP event under study but also a wide and fast shock recorded aboard both IMP-8 and Phobos-2.

\section{Modeling the particle event}

\subsection{MHD simulation of the shock propagation}

Similar to the study performed by Heras et al. (1995) we use the 2.5-dimensional MHD time-dependent code developed by $\mathrm{Wu}$ et al. (1983) to simulate the propagation of the interplanetary shock in the ecliptic plane. As described by Smith \& Dryer (1990), the initialization parameters for the MHD model are the initial disturbance speed, $V s$, at the inner boundary of the model (located at $18 R_{\odot}, 0.08 \mathrm{AU}$, from the center of the Sun); its longitudinal angular width, $\omega$; and the temporal duration of the input pulse driving time, $\tau$. In contrast to the Smith \& Dryer (1990) study, we have extended the outer boundary of the computation domain to $2 \mathrm{AU}$ and modified the prior steady-state background medium where the disturbance propagates allowing for a better description of both plasma and magnetic field observations prior to the shock arrival. We have considered that solar wind speed, density, and magnetic field magnitude at $18 R_{\odot}$ are $252 \mathrm{~km} \mathrm{~s}^{-1}$, $1197 \mathrm{~cm}^{-3}$ and $504 \mathrm{nT}$, respectively. The resulting values at 1.0 AU are $434 \mathrm{~km} \mathrm{~s}^{-1}, 4.8 \mathrm{~cm}^{-3}, 6.9 \mathrm{nT}$; and at $1.58 \mathrm{AU}$ are $435 \mathrm{~km} \mathrm{~s}^{-1}, 1.9 \mathrm{~cm}^{-3}$, and $4.0 \mathrm{nT}$, respectively, for these variables. Using the same functional form of the input pulse assumed by Smith \& Dryer (1990) we inject a pulse centered at E69 with $V s=1260 \mathrm{~km} \mathrm{~s}^{-1}, \omega=131^{\circ}$ and $\tau=1 \mathrm{~h}$. The resulting plasma disturbance reaches IMP-8 in $51.1 \mathrm{~h}\left(\langle v\rangle=813 \mathrm{~km} \mathrm{~s}^{-1}\right)$ and Phobos-2 in $77.4 \mathrm{~h}\left(\langle v\rangle=848 \mathrm{~km} \mathrm{~s}^{-1}\right)$. The transit times are measured from the onset of the X15/3B flare. Since the inner boundary of the MHD code is at $0.08 \mathrm{AU}$ from the center of the Sun, we add to the time when the MHD simulation starts the transit time spent by the input pulse to move from the solar surface to the inner boundary of the MHD code assuming that it moves at the speed $V s$.

Figure 1 shows the solar wind speed, density and magnetic field magnitude observed by IMP-8 (top panel) and Phobos-2 (bottom panel) together with the results of the simulation. The simulated shock not only reproduces the arrival time but also the jump observed in solar wind speed and density; however the simulated magnetic field shows a discontinuous jump larger than observed. The magnetic field magnitude measured by Phobos- 2 (cf. third panel of Fig. 5 of McKenna-Lawlor et al. 2005) shows periodic structures due to the spacecraft circular orbit around Mars that makes the shock identification difficult.

Figure 2 is a snapshot of the modeled shock propagation $40.7 \mathrm{~h}$ after the occurrence of the X15/3B flare. The shock front is located within the steep gradient of the density isocontours and indicated by a white arch. The locations of IMP-8 and Phobos2 are indicated by asterisks, and IMF lines are plotted as white lines. Red and orange dots indicate the points of the shock front that magnetically connect to Phobos-2 and IMP-8, respectively; each of these points is named "cobpoint" (Connecting with the OBserver POINT), after Heras et al. (1995). As the shock expands, the cobpoints of both observers scan different regions 

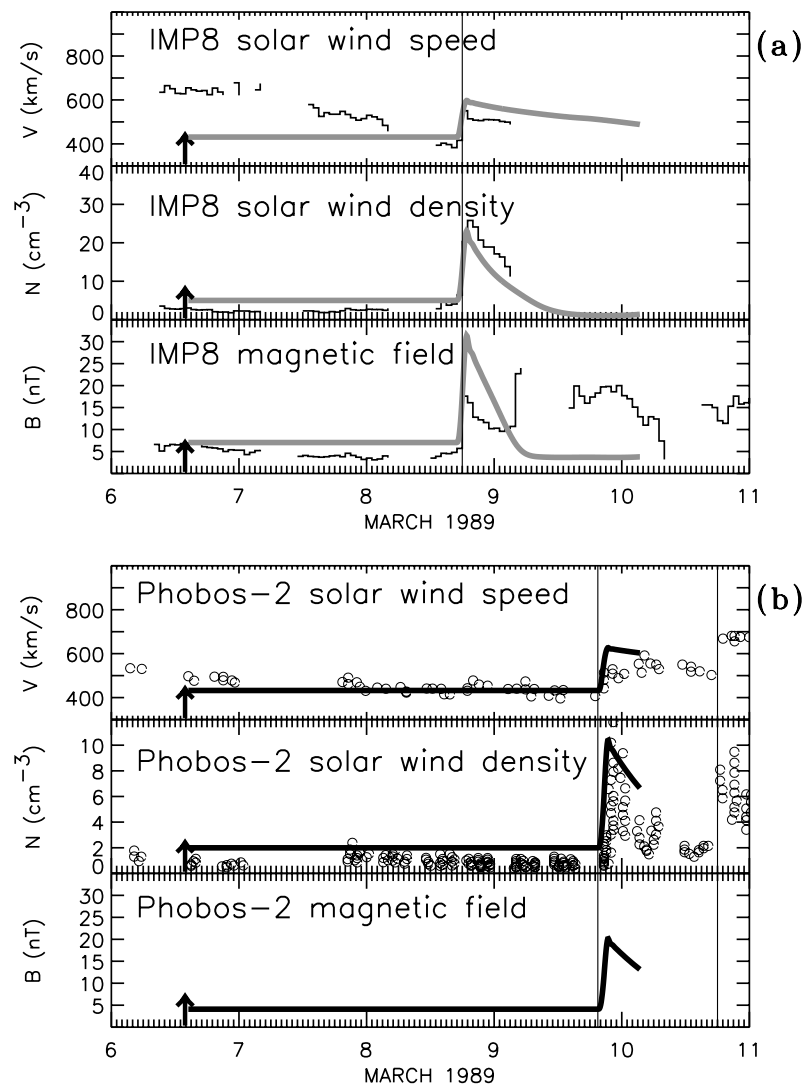

Fig. 1. (a; top panels) Solar wind speed, density and magnetic field magnitude observed by IMP-8 from 6 to 11 March and the result of the MHD shock simulation (gray line) for a hypothetic observer at the IMP-8 location. (b; bottom panels) Solar wind speed and density as measured by Phobos-2 (cf. Fig. 5 of McKenna-Lawlor et al. 2005) and the results of the MHD shock simulation (black thick line) for an observer at the Phobos-2 location. The vertical arrows indicate the onset time of the X15/3B solar flare. The first vertical lines mark the time of the shock passage as inferred from solar wind observations at each spacecraft.

of the shock front. The two top panels of Fig. 3 show the cobpoint locations versus time for both observers: panel (a) displays the heliocentric radial distance, panel (b) the azimuthal angle as measured from the Sun-IMP-8 line. Since the shock had an approximate radial expansion in all longitudes, heliocentric radial distances of the two cobpoints were very similar throughout the event (Fig. 3a); however the IMP-8 cobpoint was always more toward the west than the Phobos-2 cobpoint (Fig. 3b) at the same time.

We have also computed the downstream-to-upstream ratios of the magnetic field magnitude $B R=|B|_{(\mathrm{d})} /|B|_{(\mathrm{u})}($ Fig. 3c) and the normalized plasma velocity ratio $V R=\left(V_{r(\mathrm{~d})}-V_{r(\mathrm{u})}\right) / V_{r(\mathrm{u})}$ (Fig. 3d) at the cobpoint (where $\mathrm{u}$ and $\mathrm{d}$ stand for upstream and downstream of the shock, respectively). These parameters give a local measure of the strength of the shock at the cobpoint. Since the IMP-8 cobpoint is always more toward the western flank of the shock than the Phobos-2 cobpoint (Fig. 2), both parameters $B R$ and $V R$ are always lower for IMP-8 than for Phobos-2. In addition $B R$ and $V R$ increase with time for IMP8 , whereas for Phobos-2 they initially increase until the end of 8 March and then keep approximately constant or decrease until the shock arrival. The evolution of $V R$ and $B R$ is a consequence of (1) the cobpoint displacement along the shock front (moving from the weak western flank toward the nose of the shock), and
(2) the weakening of the shock as it propagates outward in the interplanetary medium.

Comparison of Fig. 3 with Fig. 3 of Heras et al. (1995) allows us to state that the event at Phobos- 2 has the characteristics of a central meridian event whereas the event at IMP-8 has the characteristics of an eastern event, in agreement with the heliolongitude of the X15/3B flare and the location of both observers. Vertical arrows in Fig. 3 mark the onset of the X15/3B flare and, otherwise indicated, the time origin. The time interval between these arrows and the onset of the lines is the propagation time of the shock from the Sun to the inner boundary of the MHD model, plus the time necessary for the observers to establish magnetic connection with the simulated shock. From the MHD simulation, we deduce this time to be $14.4 \mathrm{~h}$ for IMP-8 and $10.4 \mathrm{~h}$ for Phobos-2. The inner boundary of the MHD code at 0.08 AU precludes our determining of either the evolution of the actual shock from its formation closer to the Sun or its possible propagation through the solar corona (Cliver et al. 1995). The connecting time, $t_{\mathrm{c}}=21.6 \mathrm{~h}$ for IMP- 8 and $t_{\mathrm{c}}=12.6 \mathrm{~h}$ for Phobos- 2 , is determined from the time that the observer establishes magnetic connection with the part of the shock front where $V R \geq 0.1$. This is the limit where we assume that the simulated shock starts to be an efficient injector of shock-accelerated protons (Lario et al. 1998).

\subsection{Simulation of the particle event at $1 \mathrm{AU}$}

In order to reproduce the particle intensities observed by IMP-8 we have followed the procedure described by Lario et al. (1998). We assume that shock-accelerated particles are injected from the cobpoint onto the IMF line that connects the shock front with the observer. The injection rate of shock-accelerated particles is described by the function $Q(t, r)\left[\mathrm{cm}^{-6} \mathrm{~s}^{3} \mathrm{~s}^{-1}\right]$, see Eq. (1) of Lario et al. (1998). This injection rate evolves in time and space as the shock expands in the interplanetary medium and the cobpoint scans different regions of the shock front. The model assumes that $Q$ scales with energy as a power law of spectral index $\gamma: Q(E)=Q_{0}\left(E_{0}\right)\left(E / E_{0}\right)^{-\gamma}$. Here, we will consider $E_{0}=3.03 \mathrm{MeV}$, the mean energy of the $2.0-4.6 \mathrm{MeV}$ proton channel of the IMP8/CPME instrument. Energetic particles propagate along the IMF line undergoing processes of pitchangle scattering, adiabatic focusing, solar wind convection and adiabatic deceleration (Ruffolo 1995).

The pitch-angle scattering is modeled by a process of diffusion in pitch-angle described by a constant mean free path, $\lambda_{\| 0}=$ $0.6 \mathrm{AU}$, with an energy dependence given by $\lambda_{\|}=\lambda_{\| 0}\left(R / R_{0}\right)^{2-q}$ (Hasselmann \& Wibberenz 1970), where $R$ is the particle rigidity $\left(R_{0}=75.52 \mathrm{MV}\right.$, for $3.03 \mathrm{MeV}$ protons) and where $q$ is the power index of the magnetic field fluctuations spectrum (we take $q=1.5$; see, for example Kunow et al. 1991). We also consider the existence of a turbulent region 0.07 AU wide in front of the shock where particles undergo a higher frequency of pitch-angle scattering processes characterized by a mean free path $\lambda_{\| c}=0.03 \mathrm{AU}$ for $3.03 \mathrm{MeV}$ protons, that scales in rigidity as $\left(R / R_{0}\right)^{-0.8}$ (see simulations of foreshock regions in Heras et al. 1992 and Beeck \& Sanderson 1989).

Figure 4 shows the observed proton intensities in six energy channels $(0.5 \mathrm{MeV}<E<48 \mathrm{MeV})$ of the IMP-8/CPME instrument together with the fit obtained by the model, as well as the evolution of one of the computed first-order parallel anisotropies, A1/A0 (for a description of the method we use to calculate anisotropies see Sanderson et al. 1985). As can be seen in the top panel, the onset of the energetic proton intensity enhancement at $E<15 \mathrm{MeV}$ occurred before the connection 


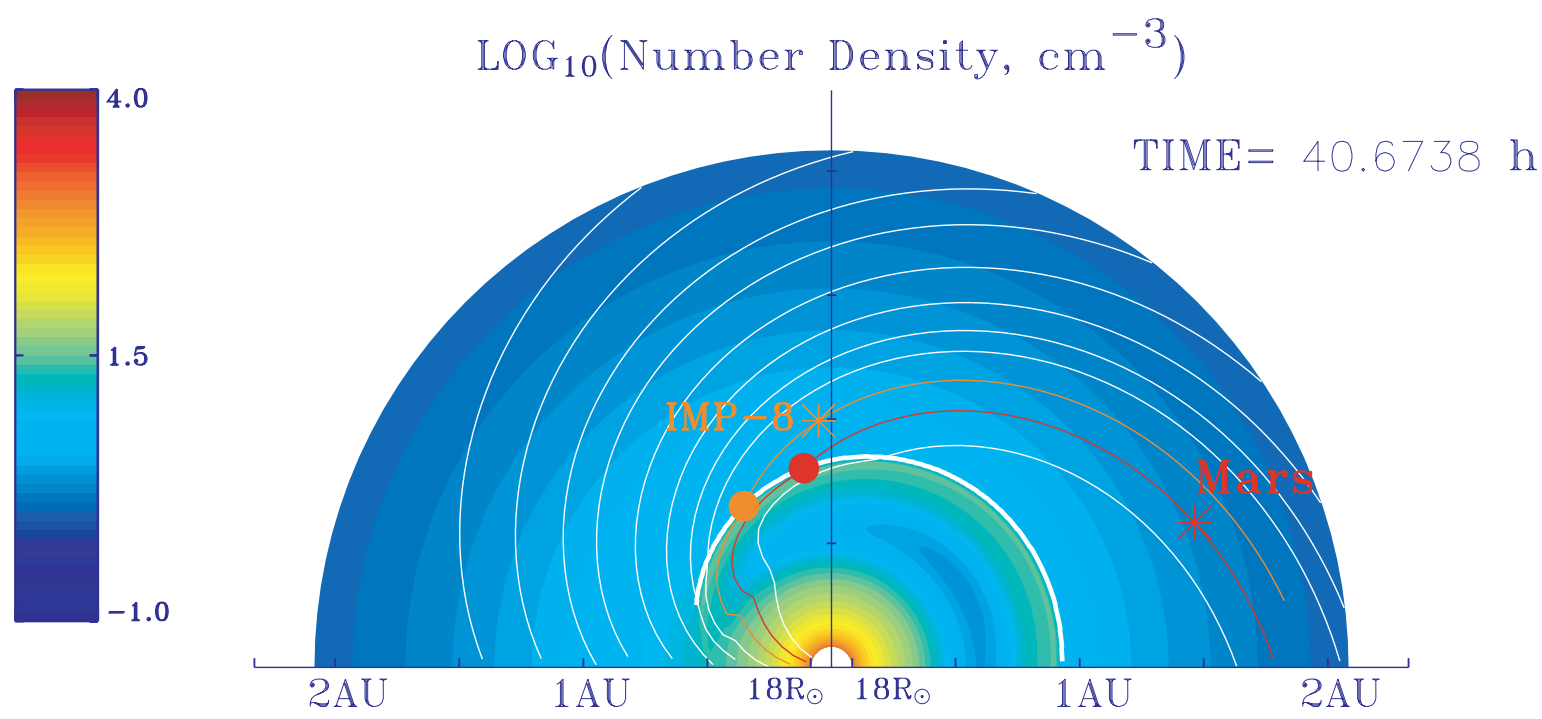

Fig. 2. Snapshot of the shock propagation simulation $40.7 \mathrm{~h}$ after the parent solar event. The density contours $\log \left(n\left[\mathrm{~cm}^{-3}\right]\right)$ and some IMF lines are represented. The locations of IMP- 8 and Mars are indicated by asterisks and their cobpoints by orange and red dots, respectively. The input pulse of the MHD shock modeling was centered at E69 (as seen from IMP-8).

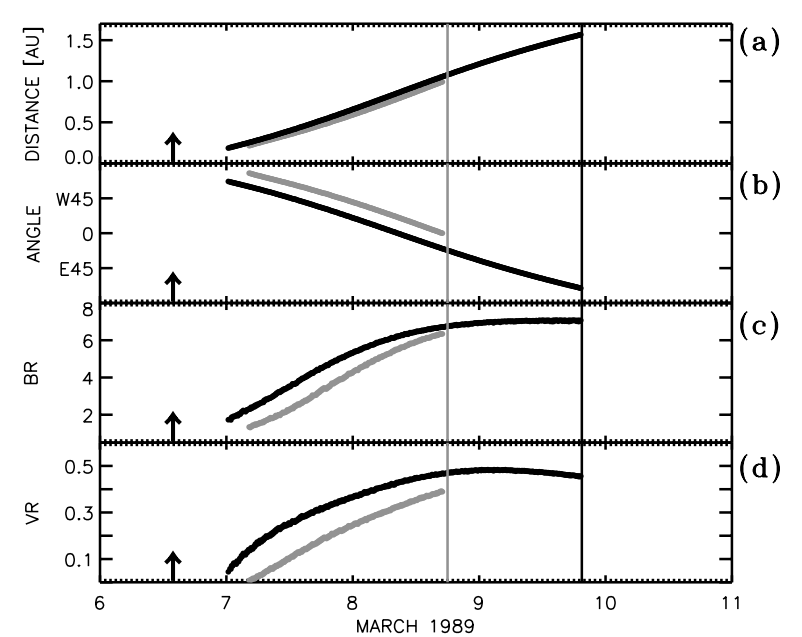

Fig. 3. Evolution of the IMP-8 (gray) and Phobos-2 (black) cobpoint; a) heliocentric radial distance, b) cobpoint heliolongitude, and parameters c) $B R$ and d) $V R$ measured at the cobpoints. The vertical arrows indicate the onset time of the X15/3B solar flare and the vertical lines the time of the shock passage as inferred from solar wind observations at IMP-8 (gray) and Phobos-2 (black).

time $t_{\mathrm{c}}$ (short solid vertical line on $7 \mathrm{March}$ ). However, this increase was delayed with respect to the time expected from the propagation of particles injected at the time of the X15/3B flare and transported along the nominal IMF Parker spiral line connected to IMP-8. For example the 4.6-15.0 MeV proton intensities started to increase at $\sim 2000$ UT on 6 March, $\sim 6 \mathrm{~h}$ after the solar flare. The increase of particle intensities at higher energies occurred presumably later than $t_{\mathrm{c}}$ since it was not observed until $\sim 1200$ UT on 7 March after a data gap, but not before 0400 UT on the same day.

The inverse velocity dispersion effect suggests that the initial particle source that magnetically connected to IMP-8 was only efficient at accelerating particles at low-energies as expected from an injection produced at the weakest part of the flank of the shock front. The inner boundary of the MHD code does not allow us to simulate the propagation of shocks in the solar corona. Therefore, we assume that particles observed before $t_{\mathrm{c}}$ are

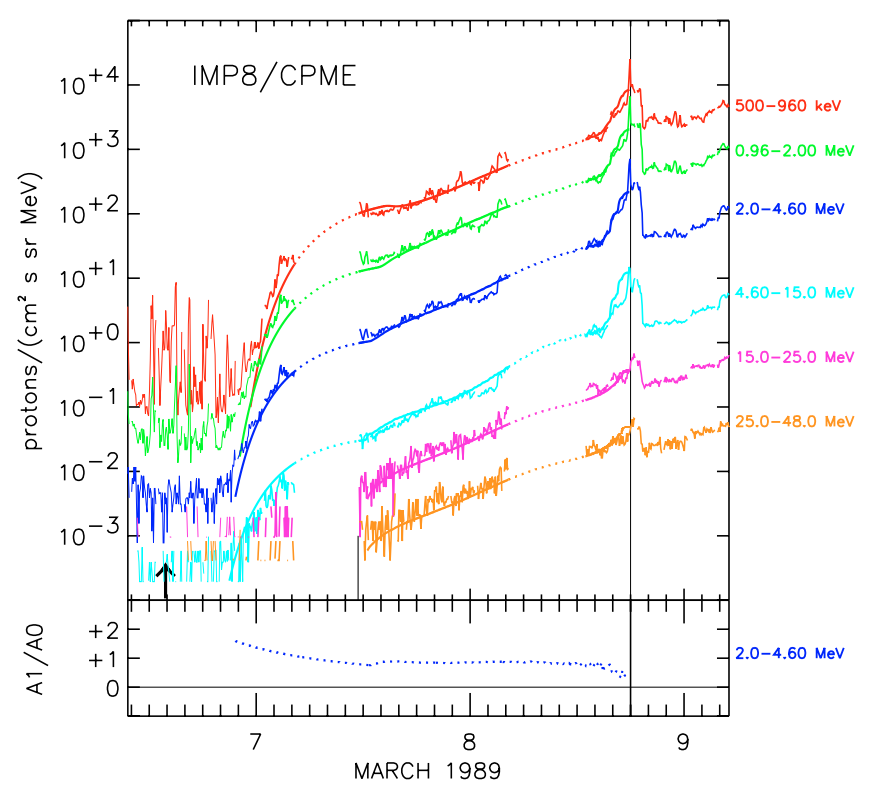

Fig. 4. Top panel: observed and simulated (lines) particle intensities for six energy channels of the IMP-8/CPME instrument, from $0.5 \mathrm{MeV}$ to $48 \mathrm{MeV}$. The arrow marks the time of the onset of the X-ray flare. The vertical line indicates the time of the shock arrival at the spacecraft and the small vertical line marks the time of connection, $t_{\mathrm{c}}$. Bottom panel: example of the evolution of the first order anisotropy parallel component, $\mathrm{A} 1 / \mathrm{A} 0$, in the upstream region, that is, from the onset of the event up to shock arrival.

accelerated by a coronal shock that only injects protons of energies below $15 \mathrm{MeV}$. The time delay between flare occurrence and particle injection onto an IMF field line connecting to IMP-8 may be interpreted as the time that the shock takes to form and to intercept at a few solar radii $\left(\sim 6 R_{\odot}\right)$ the IMF line that connects to IMP-8 (whose nominal footpoint is located at $\sim$ W53) (e.g. Mann et al. 2003). In order to reproduce the gradual release of low-energy particles from a traveling coronal shock, we assume that the particle injection before $t_{\mathrm{c}}$ depends on time as $Q \propto 1 / t \exp (-\beta / t-t / \tau)$ where $\beta=50 \mathrm{~h}$ and $\tau=15 \mathrm{~h}$ (i.e. a Reid-Axford profile, Reid 1964) and that it scales with energy as $E^{-4.3}$. 
Table 1. Spectral index, $\gamma$, of the injection rate of shock accelerated particles, $Q$, derived from the modeling of proton intensities at IMP-8.

\begin{tabular}{cccc}
\hline \hline \multicolumn{4}{c}{$\gamma$} \\
\hline Time (h) & $E \leq 1 \mathrm{MeV}$ & $1<E \leq 15 \mathrm{MeV}$ & $E>15 \mathrm{MeV}$ \\
\hline$t \leq 36.75$ & 3.4 & 4.0 & 4.0 \\
$t>36.75$ & 3.1 & 3.5 & 4.0 \\
\hline
\end{tabular}
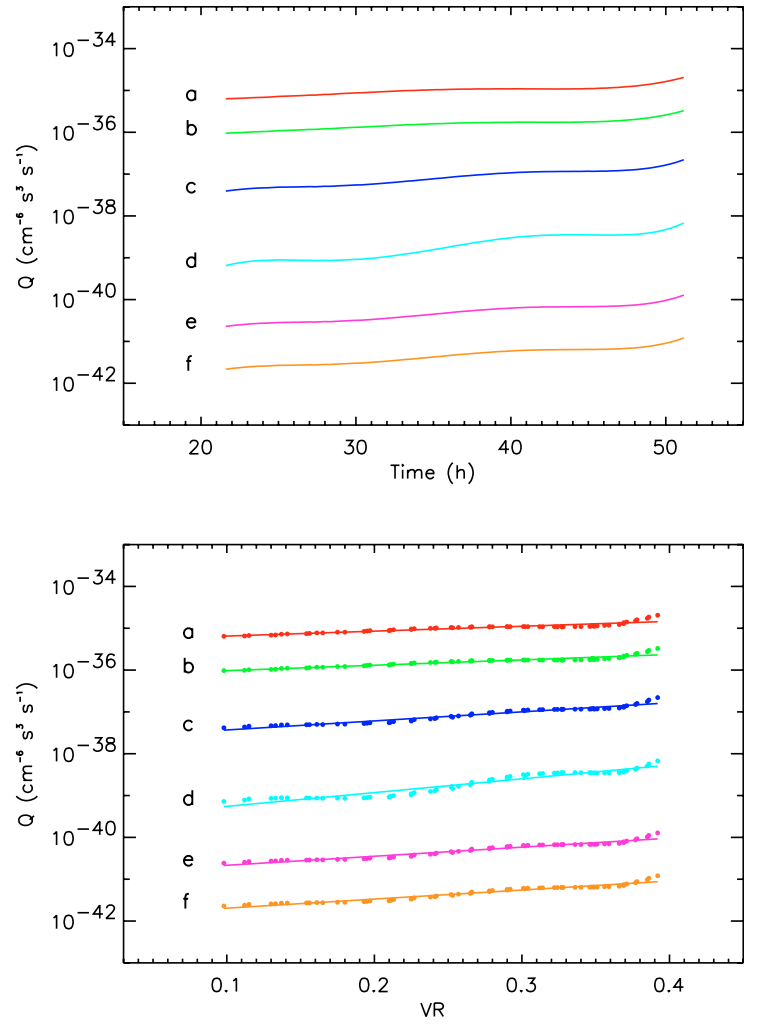

Fig. 5. Evolution of the injection rate of shock-accelerated protons, $Q$ (top panel) and correlation between $Q$ and $V R$ (bottom panel) for the six modeled energy channels (a: $0.50-0.96 \mathrm{MeV}$; b: 0.96-2.00 MeV; c: $2.0-4.6 \mathrm{MeV}$; d: $4.6-15.0 \mathrm{MeV}$; e: $15.0-25.0 \mathrm{MeV}$ and f: 25.0 $48.0 \mathrm{MeV}$ ). In the bottom panel, time runs from left to right.

The bottom panel of Fig. 4 displays the evolution of the normalized first-order parallel anisotropy; we have only plotted the case corresponding to the 2.0-4.6 MeV energy channel for clarity and because there are no anisotropy observations to compare with. Particle population evolves from a collimated distribution $(\mathrm{A} 1 / \mathrm{A} 0>1.0)$ at the onset of the event to a much less anisotropic $(<0.2)$ distribution at the shock arrival. This kind of evolution can be tracked in other eastern events (e.g. Lario et al. 1998), specially when the traveling shock continues to inject particles and the peak fluxes appear slightly after the shock passage (e.g. Sanahuja \& Domingo 1987).

The values derived for the spectral index $\gamma$ of the $Q$ function are given in Table 1 . We have considered three energy ranges, below $1 \mathrm{MeV}$, from 1 to $15 \mathrm{MeV}$ and above $15 \mathrm{MeV}$. As can be seen, the spectra become steeper with increasing energy and as longer upstream distances ahead of the shock arrival are considered (Lee 2005). The top panel of Fig. 5 shows the evolution of the injection rate of shock-accelerated particles, $Q$, for each energy channel. The cobpoint corresponding to IMP-8 slides along the shock front from the weak western flank toward the strong nose of the shock (Fig. 3), therefore $Q$ is expected to steadily increase with time. The bottom panel of Fig. 5 shows
Table 2. $Q_{0}$ and $k$ coefficients values for the $Q(V R)$ relation, $\log Q=$ $\log Q_{0}+k V R$, derived from the modeling of proton intensities at IMP-8.

\begin{tabular}{ccccc}
\hline \hline$E(\mathrm{MeV})$ & $\Delta E /\langle E\rangle$ & $Q_{0}\left(\mathrm{~cm}^{-6} \mathrm{~s}^{3} \mathrm{~s}^{-1}\right)$ & $k$ & $\xi^{a}$ \\
\hline $0.50-0.96$ & 0.66 & $4.91 \times 10^{-36}$ & 1.18 & 0.93 \\
$0.96-2.00$ & 0.72 & $7.10 \times 10^{-37}$ & 1.30 & 0.95 \\
$2.0-4.6$ & 0.86 & $2.20 \times 10^{-38}$ & 2.19 & 0.98 \\
$4.6-15.0$ & 1.25 & $2.48 \times 10^{-40}$ & 3.33 & 0.97 \\
$15.0-25.0$ & 0.52 & $1.28 \times 10^{-41}$ & 2.19 & 0.98 \\
$25.0-48.0$ & 0.66 & $1.20 \times 10^{-42}$ & 2.19 & 0.98 \\
\hline${ }^{a}$ Regression coefficient of the linear fit. & &
\end{tabular}

the correlation between $Q$ and $V R$, derived from the evolution of $Q$ (top panel) and $V R$ (bottom panel of Fig. 3); time runs from left to right. The straight line represents the fitted function $\log Q=\log Q_{0}+k V R$. This is the $Q(V R)$ relationship deduced by Lario et al. (1998). Table 2 gives the values of $Q_{0}$ and $k$, and the regression coefficient, $\xi$, obtained from each energy channel. Since $V R$ also increases with time (Fig. 3d) all the slopes, $k$, are positive.

As can be seen in Table 2, the value of $k$ derived for the 4.6-15.0 MeV channel is significantly larger than any other value deduced for the other energy channels. This fact can have consequences when predicting Phobos- 2 particle data, so, it deserves a short comment. The mean energy $(\langle E\rangle)$ of this energy channel is $8.3 \mathrm{MeV}$ and its energy window $(\Delta E)$ is $10.4 \mathrm{MeV}$. The second column of Table 2 shows that the relative width of this channel $(\Delta E /\langle E\rangle)$ is the largest of all energy channels. This is the reason why the derived value of $k$ is so high, because particle transport simulations assume $8.3 \mathrm{MeV}$ protons as representative of the entire energy channel whereas this energy channel detects protons with energies between 4.6 and $15.0 \mathrm{MeV}$. That means that we are implicitly assuming that $8.3 \mathrm{MeV}$ protons undergo the same scattering processes as the $4.6 \mathrm{MeV}$ protons or the $15.0 \mathrm{MeV}$ protons (at the foreshock, for example). In fact, when modeling such a wide 4.6-15.0 MeV channel we are forcing the shock to be more efficient at accelerating the high-energy protons of the channel than in reality it is, hence, the high value of $k$ derived.

\subsection{Modeling of the particle event at Mars}

Interplanetary conditions for energetic particle transport are usually derived from modeling SEP fluxes and first order anisotropies. As neither IMP-8/CPME nor Phobos-2/LET detectors provide particle anisotropy measurements, it is possible to derive different evolutions of the injection rate $Q$ and of the mean free path by fitting solely proton intensities. Moreover, both spacecraft are separated in radial distance $(0.58 \mathrm{AU})$ and angular position $\left(72^{\circ}\right)$; therefore, it may be that the values derived from the fitting of the corresponding fluxes at one spacecraft were different from those deduced using the other spacecraft measurements. In particular, the magnetic connection between Phobos-2 and the shock front (Fig. 3) is established earlier than with IMP8 , this means that the cobpoint of Phobos- 2 scans the shock front hours in advance than the cobpoint of IMP-8 and, therefore, under different conditions for particle acceleration. Direct modeling of the SEP event observed by Phobos- 2 can provide insights about the transport conditions and accelerated-particle injection up to the Mars orbit, within the context of the limitations of both the shock propagation and the particle transport models (Aran et al. 2004). 


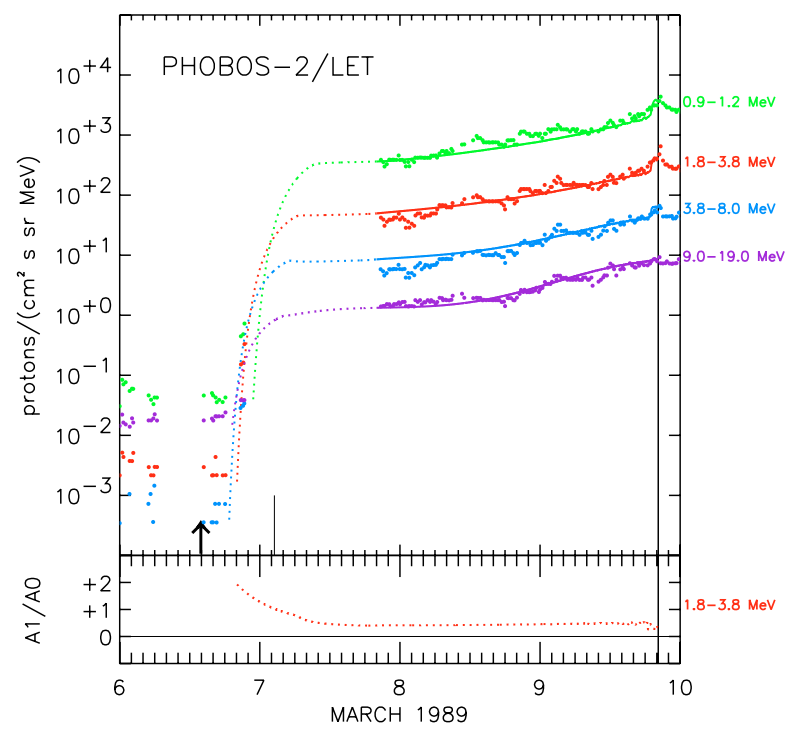

Fig. 6. Top panel: observed (small solid circles) and fitted (solid lines) particle intensities at Phobos-2/LET instrument, for four energy channels between $0.9 \mathrm{MeV}$ and $19.0 \mathrm{MeV}$ (from top to bottom: P1, P3, P4 and P5). The fitted profiles are direct output of the application of the shock-and-particle model (see text). The arrow marks the time of the onset of the X-ray flare. The vertical thick line indicates the time of the shock arrival at the spacecraft and the small vertical line marks the time of connection, $t_{\mathrm{c}}$. Bottom panel: anisotropy evolution, described as in Fig. 4.

Table 3. Spectral index, $\gamma$, of the injection rate of shock accelerated particles, $Q$, derived from the modeling of proton intensities observed at Phobos-2.

\begin{tabular}{ccc}
\hline \hline \multicolumn{3}{c}{$\gamma$} \\
\hline Time (h) & $E<1.8 \mathrm{MeV}$ & $E \geq 1.8 \mathrm{MeV}$ \\
\hline$t \leq 53.00$ & 3.1 & 3.4 \\
$t>53.00$ & 3.0 & 3.0 \\
\hline
\end{tabular}

Figure 6 shows the proton differential intensities (small solid circles) derived from the measured count rates for four (P1: 0.9-1.2 MeV, P3: 1.8-3.8 MeV, P4: 3.8-8.0 MeV and P5: $9.0-19.0 \mathrm{MeV}$ ) of the five detector channels of the Phobos-2/LET instrument (Marsden et al. 1991). We have not considered the P2 detector channel (1.2-3.0 MeV) because its energy range largely overlaps with that of $\mathrm{P} 3$. The telemetry nominal period is $20 \mathrm{~min}$. The geometric factor for P3, P4 and P5 detectors is $0.58 \mathrm{~cm}^{2}$ sr, while for P1 it is not well determined due to partial obscuration of the detector field of view by components of the spacecraft; we have assumed that P1 has a geometrical factor of $9.1 \mathrm{~cm}^{2} \mathrm{sr}$, the same as that of the identical LET instrument aboard Ulysses (Simpson et al. 1992). Under these circumstances, we have used the P1 data just to extend the energy coverage of the study.

The proton flux profiles resulting from the modeling are shown in Fig. 6 (solid and dotted lines). There is a considerable data gap at the beginning of the SEP event, from $\sim 2120$ UT on 6 March to 2045 UT on 7 March, that prevents the fitting of the earlier stages of the SEP event. The flux profile shown by dotted traces is the profile that better fits the scarce data available at the onset of the event and that later softly fits the observed and modeled flux profiles. The solid vertical line indicates the shock arrival at Phobos- 2 and the short vertical line marks when shock-accelerated particles start being injected at the Phobos-2 cobpoint $\left(t_{\mathrm{c}}=12.6 \mathrm{~h}\right)$. The magnetic connection is established closer to the Sun $\left(\sim 25 R_{\odot}\right)$ than for the IMP-8 cobpoint.

The injection rate, $Q$, derived from this fitting follows a power law; the values of the spectral index $\gamma$ are given in Table 3 . A comparison with the values of Table 1, corresponding to similar energies, shows that the energy spectrum derived at 1.6 AU is less steep (specially, above $1.8 \mathrm{MeV}$ ) than that derived at 1.0 AU. In other words, the part of shock front connected with Phobos-2 is able to inject more efficiently shock-accelerated particles at high energies than the region scanned by the cobpoint of IMP-8. This is an expected result because the SEP event is a central meridian event as seen by Phobos-2, but an eastern event as seen by IMP-8 (e.g. Heras et al. 1995).

To simulate the particle injection before $t_{\mathrm{c}}$ we have assumed a Reid-Axford time profile as for the simulation of the SEP event at IMP-8, but with $\beta=20 \mathrm{~h}$ and $\tau=15 \mathrm{~h}$ and an energy dependence of $E^{-3.4}$. Such injection allows us to fit the scarce data available at the onset of the event for the three higher energy channels (dotted traces). This initial particle injection is stronger than the one assumed for IMP-8; in fact, we should expect such behavior because the magnetic connection of Phobos- 2 with the Sun is $\sim 40^{\circ}$ closer to the center of the leading edge of the shock than that of IMP-8 (assuming a solar wind speed of $434 \mathrm{~km} \mathrm{~s}^{-1}$ and a nominal Parker spiral for the IMF). In fact it may be possible that the coronal shock traveled from its origin site (presumably close to the flare site E69) to the foot of the IMF line $\left(\sim 1.5 R_{\odot}\right)$ connecting to Phobos-2, nominally located at $\sim \mathrm{W} 13$ as seen from the Sun-Earth line (e.g. Krucker et al. 1999; Lin \& Hudson 1976).

The derived proton mean free path and its dependence on the rigidity are the same as those from the modeling of the SEP event at IMP-8. In order to reproduce the Energetic Storm Particle (ESP) component observed at $E<8 \mathrm{MeV}$, just before the shock passage, we assume a turbulent foreshock region that becomes operative 12 hours after the onset of the event $(\sim 10 \mathrm{~h}$ earlier than for IMP-8). This assumption reflects the fact that the cobpoint of Phobos-2 is located in a region of the shock front whose MHD strength is larger than the equivalent region for the IMP-8 cobpoint (displaced toward the left wing of the shock front). The particle mean free path within the foreshock is the same as that of the SEP event at IMP-8, but the dependence on the rigidity has to go as $R^{+0.2}$ to fit the flux ramp before the shock arrival (such positive values of the rigidity exponent have been derived for other SEP events, Beeck \& Sanderson 1989). The bottom panel of Fig. 6 shows the first order normalized parallel anisotropy of $2.62 \mathrm{MeV}$ protons that slowly decreases as the shock approaches the spacecraft as usually observed in western and central meridian events (Heras et al. 1994; Lario et al. 1998).

\section{Forecasting the particle event at Mars}

The injection rate $Q$ at Phobos-2 can be predicted assuming that the $Q(V R)$ relation derived from the modeling of the SEP event detected by IMP-8 (bottom panel of Fig. 5) holds throughout the entire event and all along the shock front, because the evolution of $V R$ at the Phobos-2 cobpoint is known (derived from MHD shock-modeling; Fig. 3d). Consequently, it becomes possible to synthesize particle flux profiles at $1.58 \mathrm{AU}$ and compare them with the intensity profiles observed by the Phobos-2/LET instrument. Since we cannot know a priori the transport conditions of the particles en route to Mars, in order to avoid any extra assumption, we have considered that the mean free path of the parameters characterizing the turbulent foreshock region are those obtained from the modeling of the SEP event at IMP-8. The only 
Table 4. $Q_{0}$ and $k$ coefficients values for the $Q(V R)$ relation to be applied to Phobos-2 forecasting, derived from the modeling of proton intensities at IMP-8.

\begin{tabular}{lcc}
\hline \hline$E(\mathrm{MeV})$ & $Q_{0}\left(\mathrm{~cm}^{-6} \mathrm{~s}^{3} \mathrm{~s}^{-1}\right)$ & $k$ \\
\hline P1: $0.9-1.2$ & $1.63 \times 10^{-36}$ & 1.30 \\
P3: $1.8-3.8$ & $4.25 \times 10^{-38}$ & 2.19 \\
P4: $3.8-8.0$ & $2.02 \times 10^{-39}$ & $3.33 / 2.19$ \\
P5: $9.0-19.0$ & $5.90 \times 10^{-41}$ & 3.33 \\
\hline
\end{tabular}

difference is that, because of the different magnetic connection of Phobos-2 to the Sun, the initial injection (for $t<t_{\mathrm{c}}$ ) of accelerated particles is the injection derived from the modeling at Phobos-2. As already commented, the particle injection at the earlier stages of the event is expected to be stronger for Phobos2 than that derived from the fitting of the SEP event at IMP-8.

The energy channels of the Phobos-2/LET instrument are different from those of the IMP-8/CPME instrument. Therefore, $Q_{0}$ and $k$ have to be calculated with respect to the energy channels of LET from the values derived at IMP-8 (Table 2). We assume a power law dependence with the energy for $Q_{0}$ and that the value of the slope $k$ adopted for each energy channel of the Phobos-2/LET instrument corresponds to the most similar energy channel of the IMP-8/CPME instrument.

Table 4 shows the values derived for $Q_{0}$ and $k$ for each energy. For the 3.8-8.0 MeV (P4) channel we have considered two possible choices of $k$ because this energy channel partially overlaps with the 2.0-4.6 MeV and 4.6-15.0 MeV channels of IMP8. If the dependence of particle intensity with the energy scales as a power-law, the contribution of the $3.8-4.6 \mathrm{MeV}$ protons to the intensity of the $3.8-8.0 \mathrm{MeV}$ channel is about $45 \%$. For the same reason, we have only considered the value of $k$ derived from the 4.6-15.0 MeV channel of the CPME instrument for the 9.0-19.0 MeV (P5) channel of the LET instrument; the contribution of the $9.0-15.0 \mathrm{MeV}$ protons to the intensity of the energy channel is $\sim 85 \%$.

Taking into account the aforementioned considerations we have performed three particle intensity predictions at Phobos-2, respectively named "Fc1", "Fc2" and "Fc3".

Forecast Fcl. Figure 7 displays the two predictions of the synthetic flux profiles for the 3.8-8.0 MeV channel: the dashed line profile has been computed assuming the values of $k$ corresponding to the $2.0-4.6 \mathrm{MeV}$ energy channel of IMP-8 $(k=$ $2.19)$ and the solid line that of the $4.6-15.0 \mathrm{MeV}$ channel $(k=$ 3.33). There are also two predictions for the 9.0-19.0 MeV flux profile, both calculated with the same value for $k$ (see Table 4) but assuming the absence (solid line) or presence (dotted-dashed line) of the foreshock region. The reason is that for this energy interval it is not clear whether the foreshock is active, because it partially overlaps the 4.6-15.0 MeV and the $15.0-25.0 \mathrm{MeV}$ channels of IMP-8/CPME.

As can be seen in Fig. 7, observations and synthetic profiles show good agreement $\sim 27 \mathrm{~h}$ before the shock arrival ( $\sim 15 \mathrm{~h}$ for the lowest energy channel). Just after the data gap, the computed intensities (the four solid lines) underestimate the flux observations by a factor 2.0, 2.3, 2.2 and 8.0, for channels P1, P3, P4 and P5, respectively (we call these synthetic profiles "forecast Fc1"). Several factors may be responsible for these differences:

1. The influence of the MHD conditions on the efficiency of the shock as a particle accelerator is only partially reflected in the $Q(V R)$ relation (Lario et al. 1998; Sokolov et al. 2006). It might not take into account, for example, the influence of

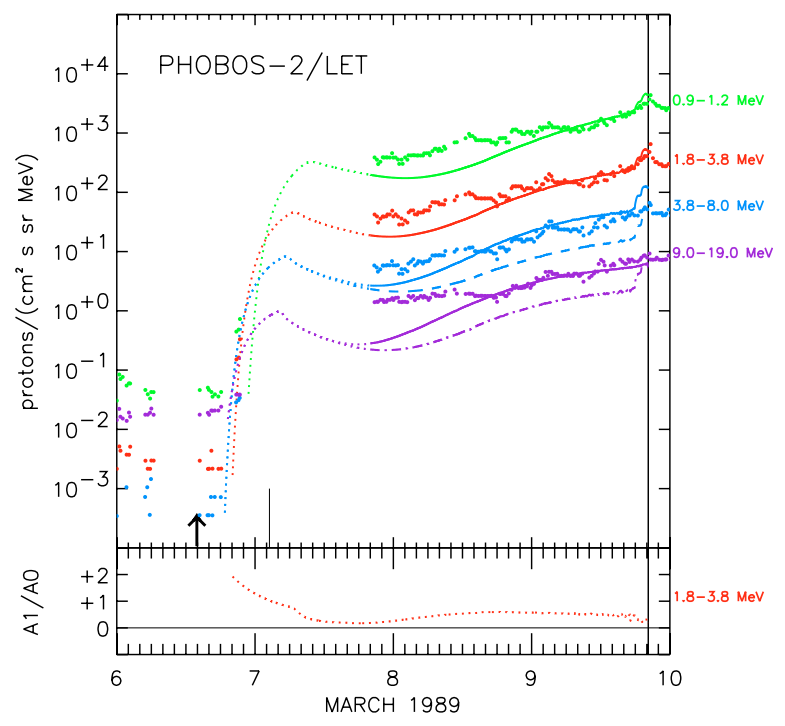

Fig. 7. Forecast Fc1. Top panel: observed (small solid circles) and predicted (solid, dashed and dotted lines) particle intensities at Phobos2/LET instrument, for the four energy channels between $0.9 \mathrm{MeV}$ and $19.0 \mathrm{MeV}$ (from top to bottom: P1, P3, P4 and P5. The two synthetic profiles (solid and dashed traces) for the 3.8-8.0 MeV channel and for the 9.0-19.0 MeV channel (solid and dotted-dashed traces) refer to different modeling conditions (see text for details). Other displayed features are as in Fig. 6.

the changes of the angle $\theta_{B n}$ as the cobpoint moves along different regions of the shock front (Tylka \& Lee 2006).

2. As the shock expands and the cobpoint moves toward the nose of the shock, the geometry of the shock may change to an oblique configuration allowing processes of particle scattering by self-generated Alfvén waves to become efficient and thus enhancing the injection rate of shock-accelerated particles (e.g. Lee 2005).

3. Particle transport conditions from the shock to IMP-8 and from the shock to Phobos-2 may be different, due to the radial and angular separation between these spacecraft.

4. The magnetic field configuration was not the nominal Parker spiral assumed here, then Phobos-2 would have been magnetically connected to locations closer to the central part of the shock front more efficient as particle-accelerators (Cane et al. 2006).

5. A local pre-existing seed particle population could make the injection rate of shock-accelerated particles more efficient at the early stages of the shock propagation from a quasiperpendicular geometry (Tylka et al. 2005).

Factor [2] assumes the formation of a foreshock in quasi-parallel or oblique shocks (Tsurutani et al. 1983; Lee 2005), although the efficiency of the particle injection does not necessarily depend on the geometry of the shock. The ESP component of the SEP event observed by IMP-8 below $\sim 15 \mathrm{MeV}$ clearly indicates that accelerated particles are trapped near the shock front at $1 \mathrm{AU}$ and thus the presence of a turbulent foreshock; the same applies with respect to the SEP event recorded at Phobos- 2 below $8 \mathrm{MeV}$ but acting earlier and probably not so strongly (see comments in the previous section). These differences can imply higher values of $Q$ at Phobos-2 than those derived from the modeling at IMP-8.

Forecast Fc2. In order to check factors [2] and [3] we have computed the proton flux profiles at Phobos- 2 by using the transport conditions derived from the modeling of the SEP event at Mars that differ from those derived at IMP-8 only by the rigidity 
dependence of the foreshock region and the time at which this region starts to act. The values of $Q_{0}$ and $k$ are the same as those used in the prediction shown in Fig. 7. The resulting protonintensity profiles ("forecast Fc2") are not shown in a figure because they are similar to those displayed in Fig. 7. They only show a slight improvement with respect to forecast Fc1 from $\sim 28 \mathrm{~h}$ before the shock passage, whereas, after the data gap, the intensities derived are underestimated by the same factors. Therefore, the possible influence of the foreshock region and the particle transport conditions acting differently on the particle population detected by IMP- 8 and Phobos- 2 cannot account for the discrepancy between observations and predictions at the beginning of the SEP event.

Forecast Fc3. Flare particle population can be an important source of seed particles in large SEP events, especially at high energies either from prior or accompanying flares (e.g. see Tylka \& Lee 2006; Cane et al. 2006). The latter could be the case, the case [5], for the present scenario. The X15/3B flare seen in conjunction with the CME on the 6 of March was located at one of the legs of the CME (Feynman \& Hundhausen 1994); then it is likely that energetic protons produced at the flare site could escape and reach the leading edge of the CME and experience further acceleration and injection there ( $\mathrm{Li} \&$ Zank 2005). Furthermore, note that an observer located at the Earth distance on the same IMF line that connects at the beginning Mars with the Sun would have seen this event as an E29 event. Although not usual, eastern events with similar longitudinal locations have exhibited a significant prompt phase of high energy (up to $400 \mathrm{MeV}$ ) protons. For instance, this is the case of the 24 September 2001 event (Lario et al. 2003b). Moreover, since IMP-8 is magnetically connected to the Sun $\sim 40^{\circ}$ westward than Phobos-2, this seed population could more easily fill the magnetic flux tubes connecting to Mars and not those (if any) to the Earth. In fact, no high energy ( $>15 \mathrm{MeV}$ ) particle fluxes were observed by IMP-8 at the onset of the SEP event. This assumption is supported by the fact that heavy-ion abundance measurements of the ESP component are consistent with the acceleration of an ambient population of solar flare particles (Marsden et al. 1991).

In order to simulate such an enhanced population of seed particles, and their presumably acceleration and injection by the shock closer to the Sun than the inner grid boundary allowed by our MHD shock simulation, we have assumed an injection of accelerated particles with a Reid-Axford profile characterized by $\beta=20 \mathrm{~h}$ and $\tau=5 \mathrm{~h}$, that scales with the energy as $E^{-2.5}$ (from $f \propto p^{-5}$, i.e. Sokolov et al. 2006). Figure 8 shows the predictions for the particle intensities obtained using such a stronger nearSun particle injection rate ("forecast Fc3"), assuming the same transport conditions and $Q(V R)$ relations as for the forecast $\mathrm{Fc} 2$.

As can be seen in Fig. 8, the predictions fit the measurements of the 1.8-3.8 MeV and 9.0-19.0 MeV channels. The two possible predictions for the 3.8-8.0 MeV channel (depending on the adopted value of $\mathrm{k}$ ) closely contour the observations. The flux prediction for the lowest energy channel has also improved with respect to forecasting Fc1 (Fig. 7) and Fc2, although it still underestimates the proton intensity from the data gap up to one day before the shock arrival. This discrepancy would disappear if a less steep spectrum were considered for the injection of low energy $(<1.8 \mathrm{MeV})$ protons near the Sun (as it happens with the injection rate of shock-accelerated particles, $Q$, derived from modeling the SEP event; see Table 3).

In spite of the gaps present in the particle data of IMP-8 and Phobos-2, and the lack of anisotropy measurements that do not permit the derivation of more accurate proton transport conditions (and hence allowing us just to assume average conditions

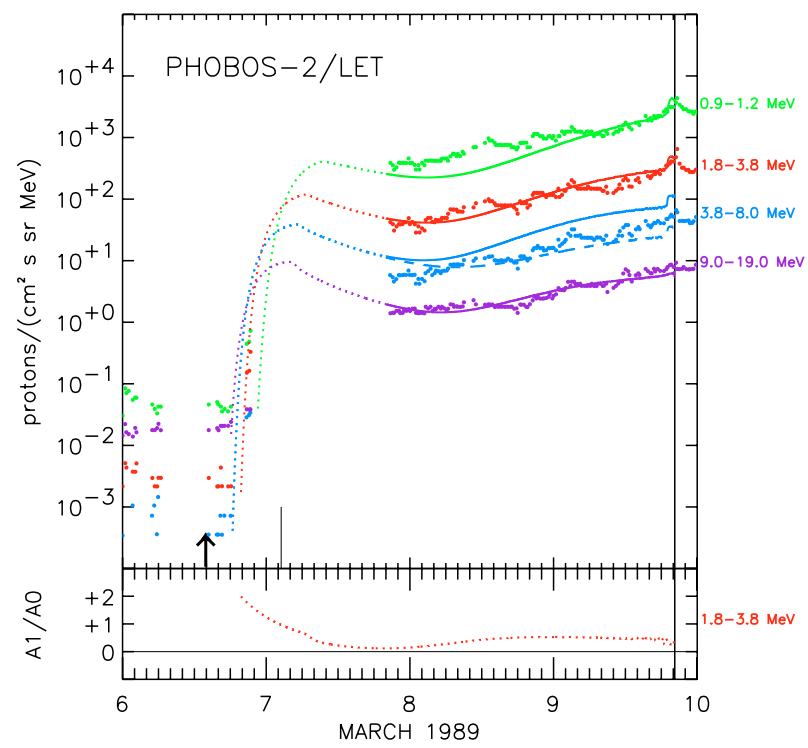

Fig. 8. Forecast Fc3. Top panel: observed (small solid circles) and predicted (solid, dotted and dashed lines) particle intensities at Phobos-2. Interplanetary propagation conditions assumed are those derived at 1.6 AU but considering a more powerful injection before the time of magnetic connection (see text for more details).

for the proton mean free path and the foreshock region), we can conclude that: (1) shock-accelerated particles en route to different observers may encounter different interplanetary transport conditions (although for this specific SEP event this effect is almost negligible); and (2) different pre-existent seed particle populations can affect differently the flux predictions of SEP events for observers in locations other than 1.0 AU.

This study is a clear example that there is not a comprehensive model able to account for all the factors at work in the generation and development of SEP events, and that SEP flux predictions out of 1.0 AU from measurements at 1.0 AU are not free of uncertainties. In fact, it would be difficult to obtain precise predictions, with absolute values in physical units of the particle intensities (relative values or predictions in arbitrary units are frequently useful enough for scientific purposes) because average values derived at 1.0 AU are not necessarily representative for other angular locations and heliocentric distances. This point can be illustrated with some numbers for the peak flux and the fluence derived from the intensity profiles fitted and forecasted for this SEP event at Mars.

\section{Fluences and peak fluxes at Mars}

In the following, "peak flux" stands for the maximum intensity of the particle directional differential intensity (or flux, as defined in ECSS E-10-04 2000). The fluence is defined here as the time integral of the differential intensity; hence, it is expressed in particles $\left(\mathrm{cm}^{2} \mathrm{sr} \mathrm{MeV}\right)^{-1}$. To compute the fluence of a SEP event for a given energy channel, we first subtract the background intensity measured before the onset of the event. Afterward, we integrate the resulting differential fluxes (either derived from observations or simulated) from the end of the initial data gap (2045 UT, March 7) up to shock arrival (2015 UT, March 9). Due to this data gap, we do not intend to derive fluence values for operational purposes but just to compare results from modeling and prediction with observations.

Table 5 lists different fluence values, for the four considered energy channels of the Phobos-2/LET instrument. The first 
Table 5. Fluences at Mars: observed, modeled and forecasted ( $\mathrm{Fc} 1, \mathrm{Fc} 2$ and $\mathrm{Fc} 3$ ) values; see text for details. Units are: $\mathrm{p}\left(\mathrm{cm}^{2} \mathrm{sr} \mathrm{MeV}\right)^{-1}$.

\begin{tabular}{lcccccc}
\hline \hline$E(\mathrm{MeV})$ & Observed & Modeled & Fc1 & Fc2 & Fc3 & \\
\hline P1: $0.9-1.2$ & 1.8 & 1.5 & 1.5 & 1.4 & 1.5 & $\left(\times 10^{8}\right)$ \\
P3: $1.8-3.8$ & 2.1 & 2.0 & 1.8 & 2.1 & 2.3 & $\left(\times 10^{7}\right)$ \\
P4: $3.8-8.0$ & 3.1 & 3.4 & 2.4 & 3.2 & 3.8 & $\left(\times 10^{6}\right)$ \\
P5: $9.0-19.0$ & 5.5 & 5.8 & 1.7 & 1.4 & 2.2 & $\left(\times 10^{5}\right)$ \\
\hline
\end{tabular}

column gives the energy range of the channel, second and third columns show the values of the fluence derived from observations and from the modeling of the flux profiles (small circles and solid lines in Fig. 6), respectively. The three following columns give the values of the fluence predicted for each case, $\mathrm{Fc} 1, \mathrm{Fc} 2$ and $\mathrm{Fc} 3$, respectively, as described in the previous section. When the model gives two predictions for the same energy channel, the average value has been calculated and listed (in italics). From the comparison between the values of the modeling of the event (Col. 3) and those derived from the measurements (Col. 2), we can conclude that the simulation fits well the observed values, slightly underestimated $(\sim 10 \%)$ for low energies $(<3.8 \mathrm{MeV})$ and slightly overestimated $(\sim 7 \%)$ at higher energies. It is worth to note that we have not tried to produce better fittings of the proton flux profiles to the observed values, for example, by assuming a radial dependence of the proton mean free path, or by trying to achieve a more precise determination of the peak flux by better tuning the injection rate spectrum.

As already commented, the data gaps, the absence of anisotropies and the small number of channels available at Phobos-2 avoid any further real improvement for prediction. As a consequence, small logarithmic differences between the output values (factors between 0.990 and 1.006, frequently enough for modeling) translate into non desirable absolute differences $(\sim 10 \%)$ for forecasting purposes. From the last three columns of Table 5 , it is easy to realize, that on average the predictions of the fluence are accurate for channels P3 and P4, correct for P1 (the observed fluence is underestimated by 17\%) and less accurate for the highest energy channel, P5 (underestimated by $70 \%$ ). When averaging over the four energy channels, the case Fc3 yields more accurate predictions than cases $\mathrm{Fc} 2$, and $\mathrm{Fc} 1$ (in spite of the fact that case $\mathrm{Fc} 3$ underestimates the observed values by a factor $\sim 0.8$ ).

Predictions globally improve when the presence of an early powerful injection of energetic particles is assumed (case Fc3). Particularly, at high energy (P5 channel), the difference with respect to observations reduces to $40 \%$ (see Table 5). This means that predictions can be improved by using a model able to simulate the shock propagation closer to the Sun (e.g. $<5 R_{\odot}$ ). Nevertheless, the discrepancy at high energy still is large (P5 energy channel in Fig. 8) because the predicted profile underestimates the observed intensity by a factor $\sim 1.4$ during the 8 hours prior to the shock arrival. This results from the fact that the predictions for the P5 energy channel are based on the values derived from modeling the 4.6-15 MeV channel of IMP-8, which has a wide energy window (see discussion in Sect. 3). Therefore, the higher energy protons detected $(\$ 15 \mathrm{MeV})$ may undergo different conditions than those of lower energies $(\gtrsim 4.6 \mathrm{MeV})$. For example, the efficiency of a shock as a particle-accelerator can start to decrease rapidly at some (unknown) energy between $\sim 5 \mathrm{MeV}$ and $20 \mathrm{MeV}$ (e.g. Tylka et al. 2000; Lario et al. 1998);
Table 6. Peak fluxes at Mars: observed, modeled and forecasted ( $\mathrm{Fc} 1$, $\mathrm{Fc} 2$ and $\mathrm{Fc} 3)$ values; see text for details. Units are: $\mathrm{p}\left(\mathrm{cm}^{2} \mathrm{sr} \mathrm{s} \mathrm{MeV}\right)^{-1}$.

\begin{tabular}{lcrrrr}
\hline \hline$E(\mathrm{MeV})$ & Observed & Modeled & Fc1 & Fc2 & Fc3 \\
\hline P1: $0.9-1.2$ & $3412.7(4361.3)^{a}$ & 3713.6 & 4266.6 & 3903.4 & 3909.0 \\
P3: $1.8-3.8$ & $443.8(650.6)^{a}$ & 400.0 & 487.0 & 451.8 & 453.4 \\
P4: $3.8-8.0$ & 66.4 & 63.9 & 64.3 & 58.4 & 58.9 \\
P5: $9.0-19.0$ & 9.2 & 8.6 & 6.8 & 6.8 & $6.8^{b}$ \\
\hline
\end{tabular}

${ }^{a}$ Values at the time of the shock passage and, between parentheses, values observed in the downstream region of the shock.

${ }^{b}$ Value at the time of the shock passage.

or the efficiency of the foreshock as temporal storage of accelerated particles can also start to diminish rapidly in the same energy range. Thus particle detectors with relatively wide energy windows in the 5-20 MeV range may not be well suited for a simultaneous and precise prediction of the fluence and the peak flux of a SEP event.

Table 6 gives the values of the peak flux measured or derived for the same cases described in Table 5. For P1 and P3 channels, the peak flux was reached 24 minutes after the time of the shock passage by Phobos-2. Our model does not allow us to simulate the downstream part of the SEP event, hence we miss the opportunity to fit the value of the peak flux for these two energy channels (these values are given between parentheses in Table 6). Instead, the observed values listed are those measured proton intensities at the shock passage. This difference is not relevant for the prediction of particle fluences $(<4 \%)$ but it is important for peak flux prediction since the flux value at the shock passage is, on average, $\sim 27 \%$ smaller than the real peak value. For channels P4 and P5 the observed peak fluxes are attained at the time of the shock passage. The peak flux given for the highest energy channel (P5) in forecast Fc3 (Table 6) is the maximum particle intensity obtained after the data gap, in order to compare it with the available data. The peak flux values derived from the modeling of the SEP event (third column) are $15 \%$ (P1 channel), $38 \%(\mathrm{P} 3), 4 \%(\mathrm{P} 4)$ and $7 \%(\mathrm{P} 5)$ smaller than the observed values. The three forecasts $(\mathrm{Fc} 1, \mathrm{Fc} 2$ and $\mathrm{Fc} 3)$ underestimate the observed peak flux values (at the shock passage) by similar percentage as the values derived from modeling, although the percentage is larger, $26 \%$, for the P5 channel (the reason is the same as that argued in the case of the fluences).

From the results shown in Tables 5 and 6, we can conclude that the differences between observational flux measurements with respect to modeled flux profiles and those differences with respect to the predicted synthetic proton flux profiles, are of the same order. Therefore, the use of the $Q(V R)$ relation allows us to predict the proton flux of the SEP event observed at Mars from the fitting to the measurements gathered at 1.0 AU of the SEP event triggered by the same interplanetary shock.

\section{Discussion and conclusions}

The dependence assumed between the injection rate of shockaccelerated particles, $Q$, and the normalized downstream-toupstream solar wind speed, $V R$, at the cobpoint (Lario et al. 1998) allows us to build up synthetic time-intensity profiles for different observers in interplanetary space. Applications of this procedure to synthesize SEP intensity profiles under different scenarios (at 1.0 AU and 0.4 AU) are under development (Aran et al. 2004, 2005, 2006). This application, called SOLPENCO 
(SOLar Particle ENgineering Code), assumes average conditions for a number of parameters (i.e. shock speed, energy spectrum of the injection rate of shock-accelerated particles, solar wind regime and IMF conditions, particle mean free path, etc.) in order to easily build up synthetic proton flux profiles. The extension of this procedure to Mars depends on the validity of such hypotheses, the most relevant being the assumption that the $Q(V R)$ relation holds for a wide variety of SEP events that may develop in a variety of solar-interplanetary scenarios (different locations of the parent solar event with respect to the observer, different shock speeds, and different conditions for particle acceleration and propagation in interplanetary space). Studies of the type presented in this work have not been regularly performed, basically because measurements of SEP events from different spacecraft at different distances and angular positions suitable to be modeled are still scarce, either closer to the Sun (Venus or Mercury orbits) or at larger (Mars orbit) heliocentric distances. In that sense, STEREO can provide data that will help us to determine the longitudinal dependences of SEP events and the dependence of $Q$ on the shock strength and geometry at two different regions of its front but not with respect to the radial distance. Up to now few works dealing with modeling SEP events observed by different spacecraft have been performed (e.g. Kallenrode 1997; Lario et al. 1998). Moreover, it is not possible to exclude that the functional dependence between the injection rate $Q$ on the MHD strength of the shock at the cobpoint can solely rely on the variable $V R$ (i.e. a dependence on $B R$ or $\theta_{B n}$ is suggested in several cases, but the MHD modeling of the shock does not allow us to draw firm conclusions).

After all these caveats that give an idea of the present limitations of any prediction, once a SEP is observed at 1.0 AU and a $Q(V R)$ relation is derived, in order to extend it to Mars, we have to be sure that the Mars orbiting spacecraft is observing the same shock as that observed at near-Earth. Unfortunately, with the present available data and the limitations for modeling, this 6 March 1989 SEP event is the only case for which we can produce such a prediction. As it can be easily understood, the Earth and Mars will not always be well located to observe the same shock, and to detect particles accelerated by the same shock but injected into the interplanetary medium from different locations ("the cobpoint" when connected to the observer) on the shock front; these are two strong constraining conditions. Furthermore, intense SEP events tend to occur in series, i.e. when a complex active group crosses the solar disk. In this case, the identification of the solar origin of each shock is not straightforward, especially at large heliocentric distances. Moreover, when a SEP event develops in the downstream region of a previous shock, the interplanetary conditions can largely differ from the assumptions commonly made by modelers. This is the reason why we applied the model only to the first event observed in the series of March 1989 events.

We have simulated both the propagation of the interplanetary shock and the time-intensity profiles observed by IMP- 8 and Phobos-2 during the event on 6 March 1989 associated with one of the most intense X-ray flares of solar cycle 22. By assuming the validity of the $Q(V R)$ relation at the cobpoint derived from the simulation of the SEP event at IMP-8, we have predicted the proton differential flux profiles observed at Phobos-2. The comparison between predicted, modeled and measured profiles at Mars yields the conclusion that the $Q(V R)$ relation performs well in forecasting the peak flux and the differential fluence at each energy channel. Unfortunately, the singularity of this SEP event prevents us from drawing any general conclusion about the validity of this assumption. We have discussed the limitations of our model and the Sun-Earth-Mars scenarios where it can provide predictions of proton flux profiles of SEP events. At present, there is no other model able to forecast proton flux profiles of SEP events at Mars from another SEP event observed at Earth, such as that presented here. We expect that future measurements of SEP events gathered by STEREO will give us the opportunity to model more events in order to evaluate the applicability of our model to forecast SEP events in the Martian environment.

Acknowledgements. The work at JHU/APL was supported under NASA grants NAG5-10787 and NAG5-13487. Work at the University of Barcelona was supported by the project AYA2004-03022 of the Spanish Ministerio de Educación y Ciencia. Partial computational support has been provided by the Centre de Supercomputació de Catalunya (CESCA). BS is member of COST 724 Action and acknowledges its support. At Exploration Physics International, Inc., and at NOAA/SEC, the work was supported by the University Partnering for Operational Support Program (UPOS) of the U.S. Department of Defense.

\section{References}

Aran, A., Sanahuja, B., \& Lario, D. 2004, Final Report. ESA/ESTEC Contract 14098/99/NL/MM

Aran, A., Sanahuja, B., \& Lario, D. 2005, Ann. Geophys., 23, 3047

Aran, A., Sanahuja, B., \& Lario, D. 2006, Adv. Space Res., 37, 1240

Balch, C. 1999, Radiation Measurements, 30, 231

Beeck, J., \& Sanderson, T. 1989, J. Geophys. Res., 94, 8769

Cane, H. V., Reames, D. V., \& von Rosenvinge, T. T. 1988, J. Geophys. Res., 93, 9555

Cane, H. V., Mewaldt, R. A., Cohen, C. M. S., \& von Rosenvinge, T. T. 2006, J. Geophys. Res., 111, 6

Cleghorn, T. F., Saganti, P. B., Zeitlin, C. J., \& Cucinotta, F. A. 2004, Adv. Space Res., 33, 2215

Cliver, E. W., Kahler, S. W., Neidig, D., et al. 1995, in Proc. 24th Int. Cosmic Ray Conf., Vol. 4, 257

Dryer, M. 1996, Sol. Phys., 169, 421

ECSS E-10-04. 2000, European Cooperation for Space Environment Standards. ESA publication

Feynman, J., \& Gabriel, S. 1988, Interplanetary Particle Environment, JPL Publication 88-28 (Pasadena, CA: JPL, California Institute of Technology)

Feynman, J., \& Hundhausen, A. J. 1994, J. Geophys. Res., 99, 8451

Gopalswamy, N., Yashiro, S., Krucker, S., Stenborg, G., \& Howard, R. A. 2004, J. Geophys. Res., 109, A12105

Hasselmann, K., \& Wibberenz, G. 1970, ApJ, 162, 1049

Heras, A. M., Sanahuja, B., Smith, Z. K., Detman, T., \& Dryer, M. 1992, ApJ, 391,359

Heras, A. M., Sanahuja, B., Sanderson, T. R., Marsden, R. G., \& Wenzel, K.-P. 1994, J. Geophys. Res., 99, 43

Heras, A. M., Sanahuja, B., Lario, D., et al. 1995, ApJ, 445, 497

Kahler, S. W. 2001, Geophys. Monogr. Ser., Origin and properties of solar energetic particles in space (Washington, DC: AGU), 125, 109

Kallenrode, M.-B. 1997, J. Geophys. Res., 102, 22347

Krucker, S., Larson, D. E., Lin, R. P., \& Thompson, B. J. 1999, ApJ, 519, 864

Kunow, S. W., Wibberenz, G., Green, G., Müller-Mellin, R., \& Kallenrode, M.-B. 1991, Energetic particles in the inner heliosphere (Berlin: Springer), 243

Kurt, V., Belov, A., Mavromichalaki, H., \& Gerontidou, M. 2004, Ann. Geophys., 22, 2255

Lanzerotti, L. 2004, Space Weather, 2, 1

Lario, D. 2005, Adv. Space Res., 36, 2279

Lario, D., \& Simnett, G. M. 2004, Geophys. Monogr. Ser., Solar particle variations (Washington, DC: AGU), 141, 195

Lario, D., Sanahuja, B., \& Heras, A. M. 1998, ApJ, 509, 415

Lario, D., Ho, G. C., Decker, R. B., et al. 2003a, in AIP Conf. Proc. Solar Wind Ten, 679, 640

Lario, D., Roelof, E. C., Decker, R. B., \& Reisenfeld, D. B. 2003b, Adv. Space Res., 32, 579

Lario, D., Kallenrode, M.-B., Decker, R. B., et al. 2006, ApJ, 653, 1531

Lee, M. A. 2005, ApJS, 158, 38

Li, G., \& Zank, G. P. 2005, Geophys. Res. Lett., 32, 2101

Lin, R. P., \& Hudson, H. S. 1976, Sol. Phys., 50, 153

Mann, G., Klassen, A., Aurass, H., \& Classen, H.-T. 2003, A\&A, 400, 329 
Marsden, R. G., Afonin, V., Erdos, G., et al. 1990, in Proc. 21st Int. Cosmic Ray Conf., 5, 121

Marsden, R. G., Wenzel, K.-P., Afonin, V. V., Gringauz, K., \& Witte, M. 1991, Planet. Space Sci., 39, 57

McKenna-Lawlor, S. M. P., Afonin, V. V., Gringauz, K. I., Keppler, E., \& Kirsch, E. 1991, Planet. Space Sci., 39, 47

McKenna-Lawlor, S. M. P., Dryer, M., Fry, C. D., et al. 2005, J. Geophys. Res., 110,3102

Reid, G. C. 1964, J. Geophys. Res., 69, 2659

Ruffolo, D. 1995, ApJ, 442, 861

Sagdeev, R. Z., \& Zakharov, A. V. 1989, Nature, 341, 58

Sanahuja, B., \& Domingo, V. 1987, J. Geophys. Res., 92, 7280

Sanderson, T. R., Reinhard, R., van Nes, P., et al. 1985, J. Geophys. Res., 90, 3973

Sarris, E. T., Krimigis, S. M., \& Armstrong, T. P. 1976, J. Geophys. Res., 81, 2341

Simpson, J. A., Anglin, J. D., Balogh, A., et al. 1992, A\&AS, 92, 365
Smart, D. F., \& Shea, M. A. 2003, Adv. Space Res., 31, 45

Smith, Z., \& Dryer, M. 1990, Sol. Phys., 129, 387

Sokolov, I. V., Roussev, I. I., Fisk, L. A., et al. 2006, ApJ, 642, L81

Tsurutani, B. T., \& Lin, R. P. 1985, J. Geophys. Res., 90, 1

Tsurutani, B. T., Smith, E. J., \& Jones, D. E. 1983, J. Geophys. Res., 88, 5645

Turner, R. 2000, IEEE Trans. on Plasma Science, 28, 2103

Tylka, A. J., \& Lee, M. A. 2006, ApJ, 646, 1319

Tylka, A. J., Boberg, P. R., McGuire, R. E., Ng, C. K., \& Reames, D. V. 2000, in Acceleration and Transport of Energetic Particles Observed in the Heliosphere, AIP Conf. Proc., 528, 147

Tylka, A. J., Cohen, C. M. S., Dietrich, W. F., et al. 2005, ApJ, 625, 474

van Nes, P., Reinhard, R., Sanderson, T. R., Wenzel, K.-P., \& Zwickl, R. D. 1984, J. Geophys. Res., 89, 2122

Watari, S., Kunitake, M., \& Watanabe, T. 2001, Sol. Phys., 204, 425

Wu, S. T., Dryer, M., \& Han, S. M. 1983, Sol. Phys., 84, 395 